\title{
Formatives Assessment
}

\author{
Elmar Souvignier • Marcus Hasselhorn
}

(C) Springer Fachmedien Wiesbaden GmbH, ein Teil von Springer Nature 2018

Das Konzept des Formativen Assessments ist in den 1960er Jahren entstanden. Nach Bloom (1969) umfasst es eine Sequenz von Maßnahmen, die aus einer Erfassung des individuellen Lernstands, einer darauf bezogenen Rückmeldung der Lernergebnisse an Lehrende und Lernende, Fördermaßnahmen sowie einem Test zur Feststellung und Dokumentation von Lernfortschritten besteht. Der Grundgedanke, Leistungsbeurteilungen zu nutzen, um Lernenden Feedback zu Stärken und Schwächen zu geben und Lehrenden diagnostische Information bereitzustellen, auf deren Basis Unterricht adaptiv gestaltet werden kann, gewann seit Mitte der 1990er Jahre zunehmend an Beachtung. Zum einen steht das Konzept unmittelbar in Einklang mit dem bildungspolitisch wie schulpraktisch relevantem Ziel individualisierter Förderung. Zum anderen beflügelten Metaanalysen (Fuchs und Fuchs 1986) und Reviews (Black und Wiliam 1998) die Überzeugung, dass die Erfassung und Rückmeldung individueller Lernfortschritte bzw. Lernverläufe sich förderlich auf die weitere Leistungsentwicklung auswirkt.

Die generell positive Einordnung des Konzepts des formativen Assessment wird in jüngeren Arbeiten differenzierter betrachtet. Dunn und Mulvenon (2009) mahnen in einem kritischen Review zunächst eine präzise Verwendung des Begriffs an, der mitunter allzu unscharf verwendet wird. Auch Kingston und Nash (2011) stellen im Rahmen einer Metaanalyse fest, dass von formativem Assessment (oder Assessment for learning) häufig bereits dann die Rede ist, wenn einzelne Komponenten (z.B.

Prof. Dr. E. Souvignier $(\bowtie)$

Institut für Psychologie in Bildung und Erziehung, Universität Münster,

Fliednerstr. 21, 48149 Münster, Deutschland

E-Mail: elmar.souvignier@uni-muenster.de

Prof. Dr. M. Hasselhorn

Bildung und Entwicklung, Deutsches Institut für Internationale Pädagogische Forschung (DIPF

Frankfurt am Main), Schloßstraße 29, 60486 Frankfurt am Main, Deutschland

E-Mail: hasselhorn@dipf.de 
Feedback) des ursprünglichen Konzepts verwendet werden. Die von ihnen ermittelten Effektstärken weisen formatives Assessment generell als wirksam aus, die Studie unterstreicht allerdings auch, dass Analysen zur Identifizierung von Wirkfaktoren und $\mathrm{zu}$ Bedingungen gelingender Implementation notwendig erscheinen. Dieses Befundmuster findet sich auch in einem Review zu Effekten von Lernverlaufsdiagnostik (Stecker et al. 2005). Hier kommt zum Ausdruck, dass in aller Regel erst die Kombination aus wiederholter diagnostischer Information und daraus resultierender differenzierter Förderung lernförderlich wirkt.

Der Grundgedanke formativen Assessments ist bestechend einfach: systematisch erhobene Leistungsinformationen werden genutzt, um pädagogische Entscheidungen mit dem Ziel einer Optimierung des Lehr-Lern-Prozesses zu treffen. Attraktiv erscheint unmittelbar, dass positive Effekte gleichsam ein Teil des Konzepts zu sein scheinen. Gleichzeitig ist das sehr allgemeine Prinzip eines data-based decision-making (Mandinach 2012) ein Rahmen, innerhalb dessen viele unterschiedliche Ansätze ihren Platz finden. So weisen Klieme et al. (2010) darauf hin, dass das Spektrum von Ansätzen, die als formatives Assessment bezeichnet werden, letztlich von adaptivem Unterricht bis hin zum ,curriculum embedded assessment“ reicht, bei dem eher formalisierte Leistungstests zum Einsatz kommen. In dem StichwortArtikel von Schütze, Souvignier und Hasselhorn werden daher zunächst begriffliche Fragen, theoretische Grundlagen und vorliegende Wirksamkeitsanalysen zusammengefasst. Zudem werden Beispiele für die Umsetzung des Ansatzes skizziert. Dabei wird deutlich, dass - in Kontrast zur scheinbaren Einfachheit des Grundgedankens formativen Assessments - die Implementation einer Sequenz aus Erfassung von Leistungsinformation, entsprechendem Feedback, Durchführung förderlicher Maßnahmen sowie der Wirksamkeitsprüfung getroffener Entscheidungen ausgesprochen herausfordernd ist.

Die drei weiteren empirischen Beiträge setzen sich mit Fragen zu Bedingungen gelingender Implementation von formativem Assessment in die schulische Praxis auseinander. Die Arbeit von Hondrich, Decristan, Hertel und Klieme stellt eine Umsetzung formativen Assessments anhand von zwei Unterrichtseinheiten im Sachunterricht der Grundschule vor, auf die Lehrkräfte anhand von Materialien und Fortbildungsveranstaltungen vorbereitet wurden. Im Zentrum der Studie steht die im Kontext formativen Assessments bislang selten adressierte Forschungsfrage nach motivationalen Effekten. Neben dem generellen Befund positiver motivationaler Effekte wird deutlich, dass das von den Kindern wahrgenommene Kompetenzerleben nach der ersten Unterrichtseinheit Effekte formativen Assessments auf die intrinsische Motivation mediiert. Gleichzeitig zeigt sich in den Unterrichtsbeobachtungen, dass Elemente formativen Assessments in geringerem Maße durch die Lehrkräfte realisiert wurden, wenn weniger unterstützende Materialien zur Verfügung gestellt wurden. In der Studie von Hebbecker und Souvignier wird ebenfalls eine Variation bei der Bereitstellung von Materialien zur Umsetzung formativen Assessments vorgenommen. Im Deutschunterricht dritter Klassen wurde Lehrkräften ein computerbasierter Ansatz zur Lernverlaufsdiagnostik zur Verfügung gestellt. Lehrkräfte in einer zweiten Bedingung erhielten zusätzliche Feedbackmaterialien und in einer dritten Untersuchungsgruppe wurde das vollständige ,Paket' aus Lernverlaufsdiagnostik, Feedback- und Fördermaterialien bereitgestellt. Während die Akzeptanz 
für die Materialien hoch ausfiel, wurde die Machbarkeit in der Unterrichtspraxis kritischer bewertet. Gegenüber der Gruppe, die nur mit der Lernverlaufsdiagnostik arbeitete, erzielten die Gruppen mit zusätzlicher Materialunterstützung keine höheren Effekte. Beide Studien, in denen konkrete Ansätze zur Umsetzung des Konzepts formativen Assessments in den schulischen Alltag untersucht wurden, unterstreichen die von Kingston und Nash (2011) hervorgehobene Notwendigkeit, jenseits der Frage nach generellen Effekten formativen Assessments vor allem Bedingungen und Herausforderungen gelingender Implementation in den Blick zu nehmen. Unter dieser Perspektive ist der Beitrag von Espin, Saab, Pat-El, Boender und van der Veen einzuordnen. Im Rahmen einer experimentellen Studie wird untersucht, ob es Merkmale bei der Darstellung von Lernverläufen gibt, die besondere Schwierigkeiten für Lehrkräfte darstellen. Ein wichtiges Ergebnis dieser Studie liegt in dem Hinweis, dass bereits die graphische Abbildung von Lernverläufen, also die Datenbasis, auf der pädagogische Entscheidungen getroffen werden, mit Interpretationsschwierigkeiten einhergehen kann. Dies gilt insbesondere dann, wenn gleichzeitig das Leistungsniveau und die Veränderungsrate in Betracht gezogen werden müssen. In der Konsequenz lässt sich aus dieser Studie ableiten, dass Fortbildungen und/ oder Interpretationshilfen sinnvoll sind, wenn das Potenzial formativen Assessments ausgeschöpft werden soll.

Das Rahmenkonzept formativen Assessments stellt zweifellos einen hochattraktiven Rahmen zur Entwicklung differenzierter Förderansätze zur Verfügung. Individuelle Lernleistungen zum Ausgangspunkt für inhaltliches Feedback und gezielte Fördermaßnahmen zu machen, bedeutet, eine optimierte Passung von Bedarf und Angebot zu realisieren. Dies entspricht letztlich auch dem von Cronbach und Snow (1977) formulierten Ansatz der Aptitude-Treatment Interaction. Das Konzept formativen Assessments bietet in diesem Sinne eine Struktur zur Umsetzung von Maßnahmen differenzierter Förderung. Mit Blick auf weitere Forschung zu formativem Assessment wird deutlich, dass experimentelle Studien und querschnittliche Analysen zu Bedingungen des Entscheidungsverhaltens auf der Basis systematisch erhobener Leistungsinformation notwendig sind, um gezielt adäquate Unterstützung bereitstellen zu können. Darüber hinaus werden Interventionsstudien benötigt, in denen konkrete Maßnahmen zur Umsetzung des Konzepts formativen Assessments praktisch erprobt und wissenschaftlich evaluiert werden.

\section{Literatur}

Black, P., \& Wiliam, D. (1998). Assessment and classroom learning. Assessment in Education: Principles, Policy \& Practice, 5, 7-74.

Bloom, B.S. (1969). Some theoretical issues relating to educational evaluation. In R. W. Taylor (Hrsg.), Educational evaluation: new roles, new means: the 68th yearbook of the National Society for the Study of Evaluation, part II (S. 26-50). Chicago: University of Chicago Press.

Cronbach, L., \& Snow, R. (1977). Aptitudes and instructional methods: a handbook for research on interactions. New York: Irvington.

Dunn, K.E., \& Mulvenon, S.W. (2009). A critical review of research on formative assessment: the limited scientific evidence of the impact of formative assessment in education. Practical Assessment, Research \& Evaluation, 14, 1-11.

Fuchs, L.S., \& Fuchs, D. (1986). Effects of systematic formative evaluation: a meta-analysis. Exceptional Children, 53, 199-208. 
Kingston, N., \& Nash, B. (2011). Formative assessment. A meta-analysis and a call for research. Educational Measurement: Issues and Practice, 30(4), 28-37.

Klieme, E., Bürgermeister, A., Harks, B., Blum, W., Leiß, D., \& Rakoczy, K. (2010). Leistungsbeurteilung und Kompetenzmodellierung im Mathematikunterricht. Zeitschrift für Pädagogik, 56, 64-74.

Mandinach, E. B. (2012). A perfect time for data use: using data-driven decision making to inform practice. Educational Psychologist, 47, 71-85.

Stecker, P. M., Fuchs, L. S., \& Fuchs, D. (2005). Using curriculum-based measurement to improve student achievement: review of research. Psychology in the Schools, 42, 795-819. 